\title{
ON A CONJECTURE OF A. J. HOFFMAN. II
}

\section{JOSEPH ZAKS}

ABstract. It is proved that certain incidence relations of hyperplanes and closed convex sets in a $d$-polytope can be preserved while replacing these sets by suitable polytopal subsets.

The purpose of this paper is to prove

THEOREM 1. If $P$ is a d-polytope in $E^{d}$ and $C_{1}, \cdots, C_{k}$ are closed convex subsets of $P$, such that every hyperplane that meets $P$ meets $\bigcup_{i=1}^{k} C_{i}$, then there exist polytopes $D_{1}, \cdots, D_{k}$ with $D_{i} \subseteq C_{i}$ for all $1 \leqq i \leqq k$, such that every hyperplane that meets $P$ meets $\bigcup_{i=1}^{k} D_{i}$.

This settles all the cases $(d, d-1, k)$, for all $d \geqq 2$ and $k \geqq 1$, of the following conjecture due to A. J. Hoffman [3]:

Conjecture $(d, t, k)$. If $P$ is a $d$-polytope in $E^{d}, d \geqq 1 ; t \geqq 0$ and $k \geqq 1$ are integers, $C_{1}, \cdots, C_{k}$ are closed convex subsets of $P$ such that every (affine) $t$-flat that meets $P$ meets $\bigcup_{i=1}^{k} C_{i}$; then there are polytopes $D_{1}, \cdots, D_{k}$ with $D_{i} \subseteq C_{i}$ for all $1 \leqq i \leqq k$, such that every $t$-flat that meets $P$ meets $\bigcup_{i=1}^{k} D_{i}$.

A. J. Hoffman proved [3] conjecture $(d, 0, k)$, for all $d \geqq 1$ and $k \geqq 1$; in these cases the $t$-flats are points and $C_{1}, \cdots, C_{k}$ cover $P$.

It follows quite elementarily that conjecture $(d, t, 1)$ is true for all $d \geqq 1$ and $t \geqq 0$, since in this case $C_{1}=P$ (see Remark 1 , here). W. R. Hare, Jr. and C. R. Smith proved [2] that conjecture $(d, t, 2)$ is true for all $d \geqq 1$ and $t \geqq 0$. We have previously shown [4] that conjecture $(d, d-2, k)$ is false for all $d \geqq 3$ and $k \geqq 4$, while here it is shown that conjecture $(d, d-1, k)$ is true for all $d \geqq 2$ and $k \geqq 1$. Conjecture $(3,1,3)$ is true (see Remark 4).

Definitions. A polytope $P$ is the convex hull of a finite set of points in the Euclidean $d$-dimensional space $E^{d}$; a $d$-polytope in $E^{d}$ is a polytope with nonempty interior; Vert $P$ denotes here the set of vertices of a polytope $P$; if $A \subset E^{d}$, conv $A$ denotes the convex hull of $A$.

An (affine) $t$-flat in $E^{d}$ is a translate of a $t$-dimensional subspace of $E^{d}$, and a hyperplane is a $(d-1)$-flat. If $H$ is a hyperplane in $E^{d}$, then $H_{+}$and $H_{-}$ ( $\stackrel{\circ}{+}_{+}$and $\stackrel{\circ}{H}_{-}$) denote the two closed (open, respectively) half-spaces of $E^{d}$, determined by $H$.

Presented to the Society, January 23, 1971 ; received by the editors October 28, 1970. AMS 1969 subject classifications. Primary 5210, 5230; Secondary 5245.

Key words and phrases. Convex $d$-polytope, hyperplane, closed convex set.

(c) American Mathematical Society 1972 
A hyperplane $H$ supports a compact set $X$ in $E^{d}$ if $X \subset H_{+}$and $H \cap X \neq \varnothing$. A hyperplane $H$ separates (strictly separates) $A$ and $B$ if $A \subset H_{+}$and $B \subset H_{-}\left(A \subset \stackrel{\circ}{H}_{+}\right.$and $B \subset \stackrel{\circ}{H}_{-}$, respectively). For additional definitions and information the reader is referred to [1].

REMARK 1. If $P, C_{1}, \cdots, C_{k}$ are as given in conjecture $(d, t, k)$, for some $d \geqq 1, k \geqq 1$ and $0 \leqq t \leqq d-1$, then Vert $P \subseteq \bigcup_{i=1}^{k} C_{i}$. To establish this, let $v \in$ Vert $P$; there exists a hyperplane $H_{v}$ of $P$ such that $H_{v} \cap P=$ $\{v\} ; H_{v}$ clearly contains a $t$-flat $F_{v}$ through $v$. Since $F_{v}$ is a $t$-flat that meets $P$ (at $v$ ), it follows by the assumptions that $F_{v}$ meets $\bigcup_{i=1}^{k} C_{i}$, i.e. $F_{v} \cap$ $\left(\bigcup_{i=1}^{k} C_{i}\right) \neq \varnothing$, and since $\bigcup_{i=1}^{k} C_{i} \subset P, F_{v} \cap\left(\bigcup_{i=1}^{k} C_{i}\right) \subset F_{v} \cap P=\{v\}$; hence $v \in \bigcup_{i=1}^{k} C_{i}$ and therefore Vert $P \subseteq \bigcup_{i=1}^{k} C_{i}$.

In case $k=1$, it follows that Vert $P \subseteq C_{1}$, and therefore $C_{1}=P$, since $C_{1}$ is a convex set contained in $P$. As a result, conjecture $(d, t, 1)$ is (trivially) true for all $d \geqq 1$ and $0 \leqq t \leqq d-1$.

We need the following:

Lemma 1. If $C$ is a convex set in $E^{d}, x \in E^{d}$, and $A$ and $B$ are such that $C=\mathrm{conv}(A \cup B)$, then every hyperplane that meets $C$ meets conv $(A \cup x) \cup$ $\operatorname{conv}(B \cup x)$.

Proof. Let $Y$ be defined by $Y=\operatorname{conv}(A \cup x) \cup \operatorname{conv}(B \cup x)$; every pair of points $y_{1}$ and $y_{2}$ of $Y$ are connected by the polyhedral path $y_{1} x \cup$ $x y_{2}$ which lies entirely in $Y$.

Let $H$ be an arbitrary hyperplane such that $H \cap C \neq \varnothing$. If $A \subset \stackrel{\circ}{H}_{+}$ and $B \subset \stackrel{\circ}{H}_{+}$, then $\operatorname{conv}(A \cup B) \subset \stackrel{\circ}{H}_{+}$, hence $C \subset \stackrel{\circ}{H}_{+}$, but this implies $C \cap H=\varnothing-$ a contradiction; therefore $A \cup B \not H_{+}$, and similarily $A \cup$ $B \nsubseteq \stackrel{\circ}{H}_{-}$. Therefore $A \cup B$ contains a point $y_{1}$ in $H_{+}$and a point $y_{2}$ in $H_{-}$; $y_{1}, y_{2} \in Y$ since $Y \supset A \cup B$. The polyhedral path in $Y$ that connects $y_{1}$ to $y_{2}$ clearly meets $H$, hence $Y \cap H \neq \varnothing$ and the proof is complete.

CoRollary 1. If $P$ is a polytope in $E^{d}, x \in E^{d}$, and Vert $P=A \cup B$, then every hyperplane that meets $P$ meets $\operatorname{conv}(A \cup x) \cup \operatorname{conv}(B \cup x)$.

Proof. In this case $P=\operatorname{conv}(\operatorname{Vert} P)=\operatorname{conv}(A \cup B)$, and Lemma 1 is applicable (with $P=C$ ).

Since the replacement of $k$ compact convex sets by polytopes can be done one at a time, we state and prove the following:

THEOREM 2. If $P$ is a d-polytope in $E^{d}, C_{1}, \cdots, C_{k}$ are closed convex subsets of $P$ such that every hyperplane that meets $P$ meets $\bigcup_{i=1}^{k} C_{i}$, then there exists a polytope $D_{1}$ in $C_{1}$ such that every hyperplane that meets $P$ meets $D_{1} \cup \bigcup_{i=2}^{k} C_{i}$.

TheOREM 3. If $P$ is a d-polytope in $E^{d}, C_{1}, \cdots, C_{k}$ are closed convex subsets of $P, C_{i} \cap C_{j}=\varnothing$ for all $1 \leqq i<j \leqq k$ and every hyperplane that meets 
$P$ meets $\bigcup_{i=1}^{k} C_{i}$, then there exists a polytope $D_{1}$ in $C_{1}$ such that every hyperplane that meets $P$ meets $D_{1} \cup \bigcup_{i=2}^{k} C_{i}$.

Clearly Theorems 1 and 2 are equivalent; our proof of Theorem 1 uses the following.

Claim 1. Theorem 3 implies Theorem 2.

Proof of Claim 1. Assuming Theorem 3 is true, we prove Theorem 2 by induction on $k$. In Case $k=1, C_{1}=P$ (by Remark 1), and one chooses $D_{1}=C_{1}$.

Assume inductively that the assertion is true for $k-1, k \geqq 2$, and let $P, C_{1}, \cdots, C_{k}$ be given as described in the statement of Theorem 2. Since the inductive assumption takes care of the cases in which $C_{i}=\varnothing$ for some $1 \leqq i \leqq k$, we assume that $C_{i} \neq \varnothing$ for all $1 \leqq i \leqq k$. If $C_{i} \cap C_{j}=\varnothing$ for all $1 \leqq i<j \leqq k$, then the existence of $D_{1}$ with the required property is guaranteed by Theorem 3, which is assumed to hold. Otherwise, let $m$ and $n$ be such that $C_{m} \cap C_{n} \neq \varnothing$ and $1 \leqq m<n \leqq k$, and let $x \in C_{m} \cap C_{n}$. Define $C_{1}^{*}, \cdots, C_{k-1}^{*}$ by

$$
C_{i}^{*}= \begin{cases}\operatorname{conv}\left(C_{m} \cup C_{n}\right) & \text { if } i=m, \\ C_{i} & \text { if } i<m \text { or } m<i<n, \\ C_{i+1} & \text { if } i \geqq n .\end{cases}
$$

Clearly, $P$ together with $C_{1}^{*}, \cdots, C_{k-1}^{*}$ satisfy all the conditions of Theorem 2 , hence the inductive assumption implies that there exists a polytope $D_{1}^{*}$ in $C_{1}^{*}$ such that every hyperplane that meets $P$ meets $D_{1}^{*} \cup \bigcup_{i=2}^{k} C_{i}^{*}$.

Case 1. $m \neq 1$. Choose $D_{1}=D_{1}^{*}$. If $H$ is a hyperplane that meets $P$, then $H$ meets $D_{1} \cup \bigcup_{i=2}^{k-1} C_{i}^{*}$, i.e. $H$ meets $D_{1} \cup \bigcup_{i \geqq 2 ; i \neq m} C_{i} \cup \operatorname{conv}\left(C_{m} \cup C_{n}\right)$. If $H$ meets $D_{1} \cup \bigcup_{i \geqq 2 ; i \neq m} C_{i}$, then clearly $H$ meets $D_{1} \cup \bigcup_{i=2}^{k} C_{i}$, as required. If $H$ meets conv $\left(C_{m} \cup C_{n}\right)$, then by Lemma $1, H$ meets $\operatorname{conv}\left(C_{m} \cup y\right) \cup \operatorname{conv}\left(C_{m} \cup y\right)$ for every $y \in E^{d}$, hence in particular $H$ meets $\operatorname{conv}\left(C_{m} \cup x\right) \cup \operatorname{conv}\left(C_{n} \cup x\right)$ (where $\left.x \in C_{m} \cap C_{n}\right)$; this means that $H$ meets $C_{m} \cup C_{n}$, because $C_{m}$ and $C_{n}$ are convex sets. Therefore $H$ meets $D_{1} \cup \bigcup_{i=2}^{k} C_{i}$, as required.

Case 2. $m=1$. In this case $D_{1}^{*} \subset C_{1}^{*}=\operatorname{conv}\left(C_{1} \cup C_{n}\right)$. Every vertex of $D_{1}^{*}$ is a finite convex combination of points of $C_{1}$ and points of $C_{2}$, therefore there exists a polytope $D_{1}^{* *}$ in $\operatorname{conv}\left(C_{1} \cup C_{n}\right)$ such that $D_{1}^{* *} \supset D_{1}^{*}$ and $\operatorname{Vert}\left(D_{1}^{* *}\right) \subset C_{1} \cup C_{n}$.

Define $D_{1}$ by $D_{1}=\operatorname{conv}\left[x \cup\left(\right.\right.$ Vert $\left.\left.D_{1}^{* *} \cap C_{1}\right)\right]$, where $x \in C_{1} \cap C_{n}$. Let $H$ be an arbitrary hyperplane that meets $P ; H$ meets $D_{1}^{*} \cup \bigcup_{i=2}^{k-1} C_{i}=$ $D_{1}^{*} \cup \bigcup_{i \geqq 2 ; i \neq n} C_{i}$, and since $D_{1}^{* *} \supset D_{1}^{*}, H$ meets $D_{1}^{* *} \cup \bigcup_{i \geqq 2 ; i \neq n} C_{i}$. If $H$ meets $\bigcup_{i \geq 2 ; i \neq n} C_{i}$, then clearly $H$ meets $D_{1} \cup \bigcup_{i=2}^{k} C_{i}$, as required. If $H$ meets $D_{1}^{* *}$, then by Corollary $1, H$ meets $\operatorname{conv}\left[x \cup\left(\right.\right.$ Vert $\left.\left.D_{1}^{* *} \cap C_{1}\right)\right] \cup$ $\operatorname{conv}\left[x \cup\left(\right.\right.$ Vert $\left.\left.D_{1}^{* *} \cap C_{n}\right)\right]$; the first set in this union is $D_{1}$, by the definition 
of $D_{1}$, and therefore if $H$ meets $D_{1}$ it clearly meets $D_{1} \cup \bigcup_{i=2}^{k} C_{i}$, as required. If, however, $H$ meets $\operatorname{conv}\left[x \cup \operatorname{Vert}\left(D_{1}^{* *} \cap C_{n}\right)\right]$, then $H$ meets $C_{n}$, since $x \in C_{1} \cap C_{n} \subset C_{n}$ and $C_{n}$ is a convex set; therefore $H$ meets $D_{1} \cup \bigcup_{i=2}^{k} C_{i}$, and Claim 1 has been established.

Proof OF Theorem 3. By induction on $k$, starting with the case $k=1$ being true by Remark 1 . Assume inductively that the assertion is true for $k-1, k \geqq 2$, and let $P, C_{1}, \cdots, C_{k}$ be given as described in the statement of Theorem 3 (assume as before that $C_{i} \neq \varnothing$ for all $1 \leqq i \leqq k$ ).

Suppose first that for some $j, 1 \leqq j \leqq k$, every hyperplane that meets $P$ meets $\bigcup_{i \neq j} C_{i}$. If $j \neq 1$, then by the inductive assumption applied to $P$, $C_{1}, \cdots, C_{j-1}, C_{j+1}, \cdots, C_{k}$, there exists a polytope $D_{1}$ in $C_{1}$, such that every hyperplane that meets $P$ meets $D_{1} \cup\left(\bigcup_{i \neq 1, j} C_{i}\right)$; therefore every hyperplane that meets $P$ meets also $D_{1} \cup \bigcup_{i=2}^{k} C_{i}$, as required. If $j=1$, then any choice for a $D_{1}$ in $C_{1}$ will do.

If however there is no such a $j$, then there are hyperplanes that meet $C_{1}$ and do not meet $\bigcup_{i=2}^{k} C_{i}$.

For every hyperplane $H$ with $H \cap C_{1} \neq \varnothing$ and $H \cap\left(\bigcup_{i \neq 1} C_{i}\right)=\varnothing$ define $K_{1}$ and $K_{2}$ by

$$
K_{1}=\operatorname{conv} \bigcup\left\{C_{i} \mid C_{i} \subset \stackrel{\circ}{H}_{+}\right\} \quad \text { and } K_{2}=\operatorname{conv} \bigcup\left\{C \mid C_{i} \subset \stackrel{\circ}{H}_{-}\right\} .
$$

Clearly $K_{1} \subset \stackrel{\circ}{H}_{+}$and $K_{2} \subset \stackrel{\circ}{H}_{-}$, hence $K_{1} \cap K_{2}=\varnothing$; not both of $K_{1}$ and $K_{2}$ are empty since $H \cap\left(\bigcup_{i \neq 1} C_{i}\right)=\varnothing, k \geqq 2$ and $C_{i} \neq \varnothing$ for all $1 \leqq i \leqq k$.

Claim 2. If $K_{1} \neq \varnothing$ and $K_{2} \neq \varnothing$, then $C$ contains a segment $L$ such that if a hyperplane $F$ separates $K_{1}$ and $K_{2}$ then $F \cap L \neq \varnothing$.

Proof. If $K_{1} \cap \operatorname{conv}\left(K_{2} \cup C_{1}\right)=\varnothing$, then $K_{1}$ and $\operatorname{conv}\left(K_{2} \cup C_{1}\right)$ can be strictly separated by a hyperplane $F_{0}$; hence $F_{0} \cap \bigcup_{i=1}^{k} C_{i}=\varnothing$; however $K_{1} \neq \varnothing, \operatorname{conv}\left(K_{2} \cup C_{1}\right) \neq \varnothing$ and $P$ being convex imply that $F_{0} \cap P \neq \varnothing$, which contradicts the assumption on $P, C_{1}, \cdots, C_{k}$. Therefore $K_{1} \cap$ $\operatorname{conv}\left(K_{2} \cup C_{1}\right) \neq \varnothing$ and similarly $K_{2} \cap \operatorname{conv}\left(K_{1} \cup C_{1}\right) \neq \varnothing$.

Take $x_{1} \in K_{1} \cap \operatorname{conv}\left(K_{2} \cup C_{1}\right)$; then there are points $y_{1} \in K_{2}$ and $z_{1} \in C_{1}$ such that $x_{1}=\lambda y_{1}+(1-\lambda) z_{1}$, for some $0 \leqq \lambda \leqq 1$; similarly take $y_{2} \in K_{2} \cap$ $\operatorname{conv}\left(K_{1} \cup C_{1}\right)$, then there are points $x_{2} \in K_{1}$ and $z_{2} \in C_{1}$ such that $y_{1}=$ $\mu x_{2}+(1-\mu) z_{2}$, for some $0 \leqq \mu \leqq 1$ (see Figure 1).

The promised segment $L$ in $C_{1}$ is taken as the segment $\left[z_{1} z_{2}\right]$. Suppose a hyperplane $F$ separates $K_{1}$ and $K_{2}$, so that say $K_{1} \subset F_{+}$and $K_{2} \subset F_{-}$. Clearly $x_{1}, x_{2} \in K_{1} \subset \stackrel{\circ}{F}_{+}$and $y_{1}, y_{2} \in K_{2} \subset \stackrel{\circ}{F}_{-}$. Since $x_{1}=\lambda y_{1}+(1-\lambda) z_{1}$ and $0 \leqq$ $\lambda \leqq 1$ it follows that $z_{1} \in F_{+}$, and similarly $y_{2}=\mu x_{2}+(1-\mu) z_{2}$ and $0 \leqq \mu \leqq 1$ imply $z_{2} \in F_{-}$. As a result $F$ meets the segment $\left[z_{1} z_{2}\right]=L$, and Claim 2 has been established.

Claim 3. If $K_{1}=\varnothing$, then $C_{1}$ contains a segment $L$ such that if a hyperplane $F$ meets $C_{1}$ and $\bigcup_{i \neq 1} C_{i} \subset \stackrel{\circ}{F}_{+}\left(\right.$or $\left.\bigcup_{i \neq 1} C_{i} \subset \stackrel{\circ}{F}_{-}\right)$, then

$$
F \cap \operatorname{conv}\left[L \cup\left(\operatorname{Ext} P \cap C_{1}\right)\right] \neq \varnothing \text {. }
$$




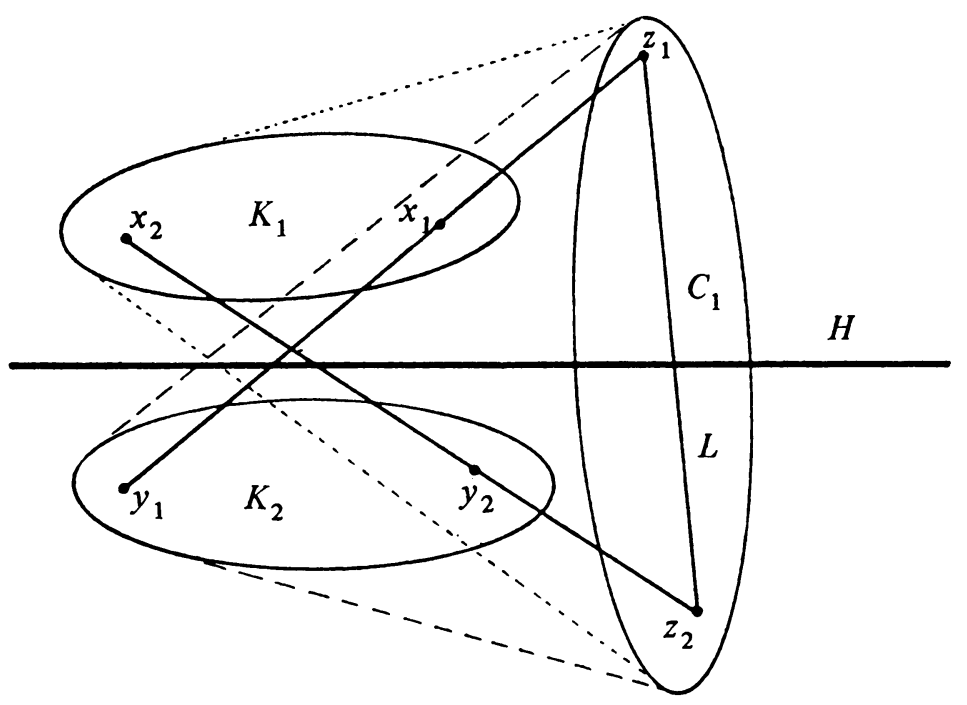

Figure 1

Proof. $\quad C_{1} \cap \operatorname{conv}\left(\bigcup_{i \neq 1} C_{i}\right) \neq \varnothing$ since otherwise $C_{1}$ and $\operatorname{conv}\left(\bigcup_{i \neq 1} C_{i}\right)$ are strictly separated by a hyperplane $F_{0}$, hence $F_{0} \cap\left(\bigcup_{i=1}^{k} C_{i}\right)=\varnothing$; since $P$ is convex it follows that $F_{0} \cap P \neq \varnothing$ which contradicts the assumption on $P, C_{1}, \cdots, C_{k}$. Let $x \in C_{1} \cap \operatorname{conv}\left(\bigcup_{i \neq 1} C_{i}\right)$, and take for the segment $L$ any segment in $C_{1}$ containing $x$ (in fact $L=\{x\}$ is as good).

Suppose $F$ is a hyperplane that meets $C_{1}$ and $\bigcup_{i \neq 1} C_{i} \subset \stackrel{\circ}{F}_{+}$; therefore $\operatorname{conv}\left(\bigcup_{i \neq 1} C_{i}\right) \subset \stackrel{\circ}{F}_{+}$and hence $x \in \stackrel{\circ}{F}_{+}$. Next $F \cap C_{1} \neq \varnothing$, hence $F_{-} \cap C_{1} \neq \varnothing$ and therefore $F_{-} \cap P \neq \varnothing$; hence $F_{-} \cap$ Ext $P \neq \varnothing$. Moreover $F_{-} \cap$ Ext $P \subset$ $C_{1}$, because Vert $P \subset \bigcup_{i=1}^{k} C_{i}$ (see Remark 1), and hence $F_{-} \cap$ Ext $P \subset$ $F_{-} \cap \bigcup_{i=1}^{k} C_{i}=F_{-} \cap C_{1} \subset C_{1}$.

We conclude that $x \in \stackrel{\circ}{F}_{+}$and $C_{1}$ contains a vertex $y$ of $P$ with $y \in F_{-}$; therefore $F \cap \operatorname{conv}\left[L \cup\left(\right.\right.$ Ext $\left.\left.P \cap C_{1}\right)\right] \neq \varnothing$.

Claim 3 has been established.

Let $L_{1}, \cdots, L_{r}$ be a collection of segments in $C_{1}$, each one obtained by applying Claims 2 and 3 to each and every different division $\{2, \cdots, k\}=$ $I \cup J$ with $I \cap J=\varnothing$, for which there exists a hyperplane $H$ with $H \cap C_{1} \neq \varnothing$, $\stackrel{\circ}{H}_{+} \supset \bigcup_{i \in I} C_{i}$ and $\stackrel{\circ}{H}_{-} \supset \bigcup_{i \in J} C_{i}$.

Define $D_{1}$ by $D_{1}=\operatorname{conv}\left\{\bigcup_{i=1}^{r} L_{i} \cup\left(\operatorname{Ext} P \cap C_{1}\right)\right]$. To show that $D_{1}$ has the required property as claimed in Theorem 3, suppose a hyperplane $H$ meets $P$. If $P \cap\left(\bigcup_{i \neq 1} C_{i}\right) \neq \varnothing$, then clearly $P \cap\left(D_{1} \cup \bigcup_{i \neq 1} C_{i}\right) \neq \varnothing$. Otherwise $P \cap\left(\bigcup_{i \neq 1} C_{i}\right)=\varnothing$, and since $H \cap P \neq \varnothing$, it follows that $H \cap$ $C_{1} \neq \varnothing$. By Claim 2 or $3, C_{1}$ contains the segment $L_{j}$ for the appropriate $j, 1 \leqq j \leqq r$, such that $H \cap \operatorname{conv}\left[L_{j} \cup\left(\right.\right.$ Ext $\left.\left.P \cap C_{1}\right)\right] \neq \varnothing$, hence $H \cap D_{1} \neq \varnothing$ as needed. $D_{1}$ is clearly a polytope in $C_{1}$.

This completes the proof of Theorem 3 . 
The proof of Theorem 1 follows now easily from the proof of Theorem 3 , which implies Theorem 2 by Claim 1, and the equivalence of Theorems 2 and 1.

REMARK 2 . Shortly before proving conjecture $(d, d-1, k)$ for all $d \geqq 2$ and $k \geqq 1$, we established conjecture $(2,1, k)$ for all $k \geqq 1$, using the following:

Claim 4. If $C_{1}$ and $C_{2}$ are disjoint compact convex sets in $E^{2}$, then they have at most four (4) common supporting lines.

Claim 5. If $C_{1}$ and $C_{2}$ are disjoint compact convex sets in $E^{d}, d \geqq 2$, $\left\{H_{i} \mid i \in I\right\}$ the collection of all the common supporting hyperplanes to $C_{1}$ and $C_{2}, x_{i} \in H_{i} \cap C_{1}$ and $y_{i} \in H_{i} \cap C_{2}$ for all $i \in I$, then every hyperplane that meets both $C_{1}$ and $C_{2}$ meets $\operatorname{conv}\left\{x_{i} \mid i \in I\right\} \cup \operatorname{conv}\left\{y_{i} \mid i \in I\right\}$.

Both Claims 4 and 5 in the case $d=2$ imply the following: "If $C_{1}$ and $C_{2}$ are disjoint compact convex sets in $E^{2}$, then there exist convex quadrangles $D_{1}$ and $D_{2}, D_{i} \subseteq C_{i}$ for $i=1,2$, such that every hyperplane that meets both $C_{1}$ and $C_{2}$ meets $D_{1} \cup D_{2}$ "'. Unfortunately, the index set $I$ in Claim 5 is infinite for all $d \geqq 3$, and there is no valid analogue of the last theorem for $E^{d}, d \geqq 3$, with "convex quadrangle" replaced by "polytopes" (take, for example, two disjoint balls).

REMARK 3. Lemma 1 can be extended as follows:

Lemma 2. If $C$ is a convex set in $E^{d}, x \in E^{d}$, and $\left\{A_{i} \mid i \in I\right\}$ are such that $C=\mathrm{conv} \bigcup\left\{A_{i} \mid i \in I\right\}$, then every hyperplane that meets $C$ meets $\bigcup_{i \in I} \operatorname{conv}\left(x \cup A_{i}\right)$.

The proof is similar to the proof of Lemma 1, hence it is omitted.

Corollary 2. If $P$ is a polytope in $E^{d}, x \in E^{d}$, and $\left\{v_{1}, \cdots, v_{n}\right\}=$ Vert $P$, then every hyperplane that meets $P$ meets $\bigcup_{i=1}^{n}\left[x, v_{i}\right]$.

Let a graph (= finite 1-dimensional simplicial complex) be called starshape if it has exactly $n+1$ vertices, one of valence $n$ and $n$ of valence 1 , $n \geqq 1$.

COROllary 3. If $P$ is a d-polytope in $E^{d}, C_{1}, \cdots, C_{k}$ are closed convex subsets of $P$, such that every hyperplane that meets $P$ meets $\bigcup_{i=1}^{k} C_{i}$, then there exist starshapes $G_{1}, \cdots, G_{k}$ with $G_{i} \subseteq C_{i}$ for all $1 \leqq i \leqq k$, such that every hyperplane that meets $P$ meets $\bigcup_{i=1}^{k} G_{i}$.

Proof. There exist, by Theorem 1 , polytopes $D_{1}, \cdots, D_{k}$ with $D_{i} \subseteq C_{i}$ for all $1 \leqq i \leqq k$, such that every hyperplane that meets $P$ meets $\bigcup_{i=1}^{k} D_{i}$; let $x_{i} \in D_{i}$, and define $G_{i}$ by $G_{i}=\bigcup\left\{\left[x, v_{i}\right] \mid v_{i} \in\right.$ Vert $\left.D_{i}\right\}$, for all $1 \leqq i \leqq k$. $G_{i}$ is a starshape, for all $1 \leqq i \leqq k$, and every hyperplane that meets $\bigcup_{i=1}^{k} D_{i}$ meets $\bigcup_{i=1}^{k} G_{i}$, by Corollary 2 . 
REMARK 4. Conjecture $(3,1,3)$ has been recently established by the author of this paper, using some ideas of [2]; the proof will appear.

REMARK 5. As stated in [3], it was M. O. Rabin who first proposed conjecture $(d, 0, k)$, for all $d \geqq 1$ and $k \geqq 1$.

\section{REFERENCES}

1. B. Grünbaum, Convex polytopes, Pure and Appl. Math., vol. 16, Wiley, New York, 1967. MR 37 \#2085.

2. W. R. Hare, Jr. and C. R. Smith, On convex subsets of a polytope, Proc. Amer. Math. Soc. (to appear).

3. A. J. Hoffman, On the covering of polyhedra by polyhedra, Proc. Amer. Math. Soc. 23 (1969), 123-126. MR 40 \#835.

4. J. Zaks, On a conjecture of A. J. Hoffman, Proc. Amer. Math. Soc. 27 (1971), 122125.

Department of Mathematics, Michigan State University, East Lansing, MiCHIGAN 48823

Current address: Department of Mathematics, University of Haifa, Haifa, Israel 